\title{
Los Angeles Homelessness and the Access to Water, Sanitation, and Hygiene
}

\author{
Lourdes Johanna Avelar ${ }^{\mathrm{a},}$ *, Yao-Yi Chiang ${ }^{\mathrm{b}}$, Robert O. Vos ${ }^{\mathrm{c}}$, Jose Jesus Rico ${ }^{\mathrm{d}}$, Yanyi Qian ${ }^{\mathrm{e}}$, \\ Xiaozhe Yin ${ }^{\mathrm{f}}$, Kate Vavra-Musser ${ }^{\mathrm{g}}$ \\ ${ }^{a}$ Spatial Sciences Institute, University of Southern California, USA, avelarpo@usc.edu \\ ${ }^{b}$ Spatial Sciences Institute, University of Southern California, USA, yaoyic@usc.edu \\ cS Spatial Sciences Institute, University of Southern California, USA, vos@usc.edu \\ ${ }^{d}$ Spatial Sciences Institute, University of Southern California, USA, joserico@usc.edu \\ e Department of Information Management, Peking University, China, erica_qian@pku.edu.cn \\ ${ }^{f}$ Spatial Sciences Institute, University of Southern California, USA, xiaozhey@usc.edu \\ ${ }^{g}$ Spatial Sciences Institute, University of Southern California, USA, vavramus@usc.edu \\ * Corresponding author
}

Keywords: Homelessness, Water, WaSH, Water Insecurity, Los Angeles

\begin{abstract}
:
Homelessness is a problem increasingly visible in many urban and rural communities that have rendered these marginalized people invisible. In Los Angeles County, approximately 53,000 people experience homelessness in a single-night, 4,294 of which reside in downtown's "Skid Row" (LASHA, 2018). In the past months, cases of hepatitis A and murine typhus outbreaks have been reported in Skid Row. These infectious diseases are associated with poor living environments and inadequate access to basic water, sanitation, and hygiene services (WaSH). Therefore, this study addresses homelessness using water insecurity as a lens to look beyond the provisioning of housing (a long-term goal). More specifically, it examines the consequences of water insecurity in the health and lives of people experiencing homelessness using 134 in-depth interviews and surveys collected in July-August 2018. The data we collected were abstracted and analysed in $\mathrm{R}$ studio to test for associations between differences in poor access to $\mathrm{WaSH}$ and demographic factors, and then visualized in ArcGIS Pro using Hot Spot analysis. The study findings highlight that 56\% of all study participants reported difficulty accessing basic WaSH services. Of this total, 65\% of women participants reported statistically significant differences in access to showers compared to men $(p<0.05)$. Additionally, racial differences were reported in the sample, with Hispanic/Latinx and mixed-racial groups having more difficulty in accessing laundry services, drinking water, and showers. Furthermore, temporal differences existed in access to WaSH services, with people resorting to use public spaces (sidewalks, alleyways, and buckets inside their tents) more often at night than morning-time, due to a lack of public facilities remaining open and available throughout the day. Since the data collection took place in the summer time, $43 \%$ of participants reported experiencing dehydration, but also diarrhoea (24.39\%), and skin infections (20.33\%), all which are attributed to their reduced access to WaSH services. Overall, the information gathered from this study provides a deeper understanding of the existing inequities in access to services. It also demonstrates that there is a need for better public policies to address homelessness with more equitable solutions including gender, race, age, and immigration status. In conclusion, increasing access to WaSH resources can be a form of an immediate intervention provided to the homeless in order to 1) restore their human dignity, 2) improve their living environments, which would then lead to 3) the reduction of future health outbreaks and cost of medical emergency services.
\end{abstract}

